\title{
The effects of exopolysaccharides and exopolysaccharide-producing Lactobacillus on the intestinal microbiome of zebrafish (Danio rerio)
}

Chenchen Ma ${ }^{1+}$, Hongyang Guo ${ }^{1,2+}$, Haibo Chang ${ }^{1}$, Shi Huang ${ }^{3}$, Shuaiming Jiang ${ }^{1}$, Dongxue Huo ${ }^{1}$, Jiachao Zhang ${ }^{1 *}$ and Xiaopeng Zhu ${ }^{1,2^{*}}$ (D)

\begin{abstract}
Background: Numerous studies have reported the health-promoting effects of exopolysaccharides (EPSs) in in vitro models; however, a functional evaluation of EPSs will provide additional knowledge of EPS-microbe interactions by in vivo intestinal microbial model. In the present study, high-throughput amplicon sequencing, short-chain fatty acid (SCFAs) and intestinal inflammation evaluation were performed to explore the potential benefits of exopolysaccharides (EPSs) and EPS-producing Lactobacillus (HNUB20 group) using the healthy zebrafish (Danio rerio) model.

Results: The results based on microbial taxonomic analysis revealed that the abundance of four genera, Ochrobactrum, Sediminibacterium, Sphingomonas and Sphingobium, were increased in the control group in comparison to HNUB20 group. Pelomonas spp. levels were significantly higher and that of the genera Lactobacillus and Brachybacterium were significantly decreased in EPS group compared with control group. PICRUSt based functional prediction of gut microbiota metabolic pathways indicated that significantly lower abundance was found for transcription, and membrane transport, whereas folding, sorting and degradation and energy metabolism had significantly higher abundance after HNUB20 treatment. Two metabolic pathways, including metabolism and endocrine functions, were more abundant in the EPS group than control group. Similar to the HNUB20 group, transcription was also decreased in the EPS group compared with the control group. However, SCFAs and immune indexes indicated EPS and HNUB20 performed limited efficacy in the healthy zebrafish.

Conclusions: The present intestinal microbial model-based study indicated that EPSS and high-yield EPSproducing Lactobacillus can shake the structure of intestinal microbiota, but cannot change SCFAs presence and intestinal inflammation.
\end{abstract}

Keywords: Exopolysaccharides, Intestinal microbiota, Zebrafish, Lactobacillus, Intestinal inflammation, Short-chain fatty acid

\footnotetext{
* Correspondence: zhjch321123@163.com; biozxp@163.com

${ }^{+}$Chenchen Ma and Hongyang Guo contributed equally to this work.

${ }^{1}$ College of Food Science and Engineering, School of Life and

Pharmaceutical Sciences, Hainan University, Haikou 570228, Hainan, P. R.

China

Full list of author information is available at the end of the article
}

(c) The Author(s). 2020 Open Access This article is licensed under a Creative Commons Attribution 4.0 International License, which permits use, sharing, adaptation, distribution and reproduction in any medium or format, as long as you give appropriate credit to the original author(s) and the source, provide a link to the Creative Commons licence, and indicate if changes were made. The images or other third party material in this article are included in the article's Creative Commons licence, unless indicated otherwise in a credit line to the material. If material is not included in the article's Creative Commons licence and your intended use is not permitted by statutory regulation or exceeds the permitted use, you will need to obtain permission directly from the copyright holder. To view a copy of this licence, visit http://creativecommons.org/licenses/by/4.0/ The Creative Commons Public Domain Dedication waiver (http://creativecommons.org/publicdomain/zero/1.0/) applies to the data made available in this article, unless otherwise stated in a credit line to the data. 


\section{Background}

Lactobacillus and Bifidobacterium spp, have been widely used as probiotics to prevent disease and improve host health via the gut microbiota [1, 2]. Exopolysaccharides (EPSs) are carbohydrate polymers composed of monosaccharides, including homopolysaccharides and heteropolysaccharides, and are secreted by many bacteria [3, 4]. In particular, beneficial health effects of EPSs produced by lactic acid bacteria (LABs) have been focused [5]. The human gut is inhabited by a large number of bacteria, which are involved in maintenance of host homeostasis. As a potential prebiotic, EPSs are also a source of energy and nutrients available to the gut microbiota [6]. Lactobacillus plantarum and Bifidobacterium derived EPSs can increase the content of shortchain fatty acids (SCFAs) in faeces [7, 8]. Previous study reported that SCFAs are produced by gut microbiota as products of dietary fiber fermentation and may support host antibody responses [9]. EPS from Lactobacillus plantarum NCU116 can regulate intestinal epithelial barrier function [10]. Furthermore, EPS of Lactobacillus paraplantarum BGCG11 reduces inflammatory hyperalgesia in rats [11]. In addition, an anti-obesity effect in mice [12], promotion of probiotic bacteria growth [13] and a cholesterol-lowering effect in mice [14] have also been reported. Notably, EPSs also enhanced the growth of Clostridium in vitro [15]. Nonetheless, it remains unclear whether EPSs are actually beneficial to the host. Thus, in vivo studies are urgently needed to explore the interaction among EPSs, EPS-producing strains and the gut microbiota to confirm or refute the capability of LAB EPSs to exert a healthy effect on the host.

In recent decades, use of zebrafish (Danio rerio) as a model system to study the correlation of gut microbiota and host health with bacterial exposure has expanded due to the high conservation of genes between zebrafish and humans, with $87 \%$ conservation overall [16]. Their intestines are small, facilitating the study of the entire intestinal microbiota $[17,18]$. Given the highly conserved nature of innate defense in zebrafish, it represents an ideal model system for inflammatory bowel disease [19]. An EPS-producing bacteria have shown positive health properties in zebrafish models [20]. A previous study has shown that Lactobacillus spp. may colonize the intestinal tract of zebrafish [21]. Nevertheless, evaluation of the health benefits of Lactobacillus EPS and EPS-producing Lactobacillus in a zebrafish adult model has remained unexplored.

Thus, to further understand the effect of EPS and EPS-producing strains in the healthy zebrafish, we performed preliminary research investigating the gut microbiota, microbiota metabolic pathways, SCFAs presence and intestinal inflammation. The results of this study provide novel data on health benefits of Lactobacilus and Lactobacillus derived EPS, and we highlight the fact that probiotics and prebiotics need more evaluation in vivo using animal models.

\section{Results \\ Bacterial community richness and diversity in healthy zebrafish}

Chao1 and ACE indices represent the community richness, and Shannon and Simpson indices represent community diversity. We compared ACE, Chao1, Shannon, and Simpson indices in day seven. EPS compared to control group, there were no significant differences $(P$ values $>0.05$, by Wilcoxon rank sum test) in all indices. Chao1 index in HNUB20 group was lower than that in the control group, while no significant differences were found in other indices (Table 1).

\section{Bacterial community composition and comparison of different genera}

The core genera were defined as those present in carried at least by $80 \%$ of samples, and with average relative abundance at $1 \%$ [22]. Changes in the core microbiota, including Proteobacteria (Acinetobacter, Aeromonas, Ochrobactrum, Pelomonas, Ralstonia, Sphingomonas and Vibrio), Fusobacteria (Cetobacterium), Bacteroidetes (Sediminibacterium) of the zebrafish intestine are presented as histograms in Fig. 1a-c. Pelomonas and Ralstonia maintained the same variation trend (increase, decrease, increase) during 7 days of HNUB20 and EPS treatment, as did Cetobacterium (increase, increase, decrease). We compared the intestinal structure based on PCoA of weighted UniFrac distances (Fig. 1d-f), and the results revealed dynamic changes in the microbiota over time among the three groups. We found the intestinal microbiota of the EPS and HNUB20 groups were most stable compared with the control group. The abundance of bacteria in phyla level and core genera at 7 days could be found in more detail (see Additional file 6 and Additional file 7).

To analyse the beta diversity of different groups, Weighted UniFrac distances and Adonis analysis were performed for day 7 samples. The results showed that the differences in microbial structure among the three groups were significant and distinct, with a $P$ value of 0.047 , a $R$ value of 0.2475 (Fig. 1 g). Furthermore, the structural similarity of the intestinal microbiota between the EPS and control groups and the L. fermentum HNUB20 and control groups were compared, and the results are shown in Fig. $1 \mathrm{~h}$. The similarity between the EPS and the control groups was higher than that between the L. fermentum HNUB20 and control groups, indicating that EPS had a lesser effect on the intestinal microbial community than did strain HNUB20. 
Table 1 Comparison of alpha diversities among three groups

\begin{tabular}{llllll}
\hline & $\begin{array}{l}\text { HNUB20 } \\
(\text { mean } \pm \text { SD) }\end{array}$ & $\begin{array}{l}\text { Con } \\
(\text { mean } \pm \text { SD) }\end{array}$ & $\begin{array}{l}\text { EPS } \\
(\text { mean } \pm \text { SD) }\end{array}$ & $\begin{array}{l}\boldsymbol{P} \text { value } \\
\text { (B20 vs Con) }\end{array}$ & $\begin{array}{l}\boldsymbol{P} \text { value } \\
\text { (EPS vs Con) }\end{array}$ \\
\hline Simpson & $0.86 \pm 0.04$ & $0.91 \pm 0.03$ & $0.88 \pm 0.01$ & 0.095 & 0.31 \\
Chao1 & $660.14 \pm 178.33$ & $701.20 \pm 182.64$ & $647.12 \pm 137.88$ & 0.008 & 0.548 \\
ACE & $680.19 \pm 201.47$ & $711.82 \pm 165.30$ & $652.21 \pm 146.32$ & 0.814 & 0.691 \\
Shannon & $4.66 \pm 0.57$ & $5.46 \pm 0.69$ & $4.96 \pm 0.13$ & 0.222 & 0.548 \\
\hline
\end{tabular}

Next, different intestinal microbiota genera (relative abundance $>1 \%, P<0.01$ by the Wilcoxon rank sum test) were compared, as shown by a heatmap in Fig. 1i, in which the stars represent different genera. Four different genera, Ochrobactrum, Sediminibacterium, Sphingomonas and Sphingobium, showed significantly higher abundance in the control group versus the HNUB20treated group. In addition, Pelomonas was present at significantly higher abundance in the EPS group than in the control group, and Lactobacillus and Brachybacterium were in higher abundance in the control group than in the EPS group.

\section{Comparison of microbial metabolic pathways predicted by PICRUSt}

To better understand differences in microbial functions among the three groups, functional features were predicted by PICRUSt for day 7 samples. The abundance of metabolic pathways in level 2 based on KEGG database could be found in more detail (see Additional file 8). First, we performed PCA analysis based on metabolic pathway relative abundance in level 2; second, Adonis analysis was performed to calculate differences among the three groups. The clusters with a $P$ value of 0.012 , a $\mathrm{R}$ value of 1.827 are shown in Fig. 2a, and the different metabolic pathways are shown (relative abundance $>1 \%, P<0.01$, by the Wilcoxon rank sum test) using a boxplot (Fig. 2b-c). Compared with the control group, the HNUB20 group exhibited five differentially enriched metabolic pathways: significantly lower abundance was found for transcription, and membrane transport, whereas folding, sorting and degradation and energy metabolism had significantly higher abundance. Two metabolic pathways, including metabolism and endocrine functions, were more abundant in the EPS group than in the control group. Similar to the HNUB20 group, transcription was also decreased in the EPS group compared with the control group. Therefore, the functional structure was changed by consuming EPS and L. fermentum HNUB20 for 7 days.

\section{The impacts of EPS and HNUB20 on the intestinal SCFAs} We detected SCFAs of the whole zebrafish intestine, including acetic acid, propionic acid, butyric acid, valeric acid and n-hexanoic acid. In the healthy zebrafish (Fig. 3), the abundance of acetic acid and propionic acid showed no significant difference by EPS and HNUB20 treatment. Compared with control group, n-hexanoic acid were significantly higher than EPS group. An increase in butyric acid was observed by HNUB20 treatment. The results indicated EPS and HNUB20 had limited influence on SCFAs abudance of the whole zebrafish intestines. Here, although, we did not accurately describe the content of SCFAs produced by the intestinal microbiota. Our PICRUSt results showed that there was no difference in the citrate cycle (TCA cycle) among the three groups (see Additional file 4), and there may be no difference in the consumption of SCFAs. Therefore, it was feasible to determine the SCFA of the whole intestine according to previous research [23].

\section{Immune indexes response to EPS and EPS producing lactobacillus comsumption}

Key inflammatory mediators or immunoglobulin IL-4, IL-10, sIgA were detected by ELISA, no significant differences were observed in the healthy zebrafish among all indexes (Fig. 4).

\section{The correlation network revealed the potential} modulation process of EPS and Lactobacillus fermentum HNUB20 in the healthy zebrafish

Differentially abundant genera and metabolic pathways were revealed by the above analysis in the healthy zebrafish. To better show correlations between the differentially abundant genera and metabolic pathways, Spearman's correlation coefficient was calculated based on the relative abundance of different genera, SCFAs, immune indexes and metabolic pathways. We then performed two network analyses to explore the dynamic processes occurring when zebrafish consume EPS and L. fermentum HNUB20 (Fig. 5). The network showed high correlations among strain HNUB20, differentially abundant genera, SCFAs, immune indexes and metabolic pathways. It could be observed the intake of HNUB20 or EPS affected intestinal microbiota and metabolic profiles. Meanwhile SCFAs stimulated the host's immune response, accompanied with the rise or fall of immune indexes including sIgA, IL-4 and IL-10. Although limited effects were found by EPS and EPS-producing strain in this study, the potential correlation network may show that the EPS and HNUB20drived changes in intestinal microbiota and the gut 


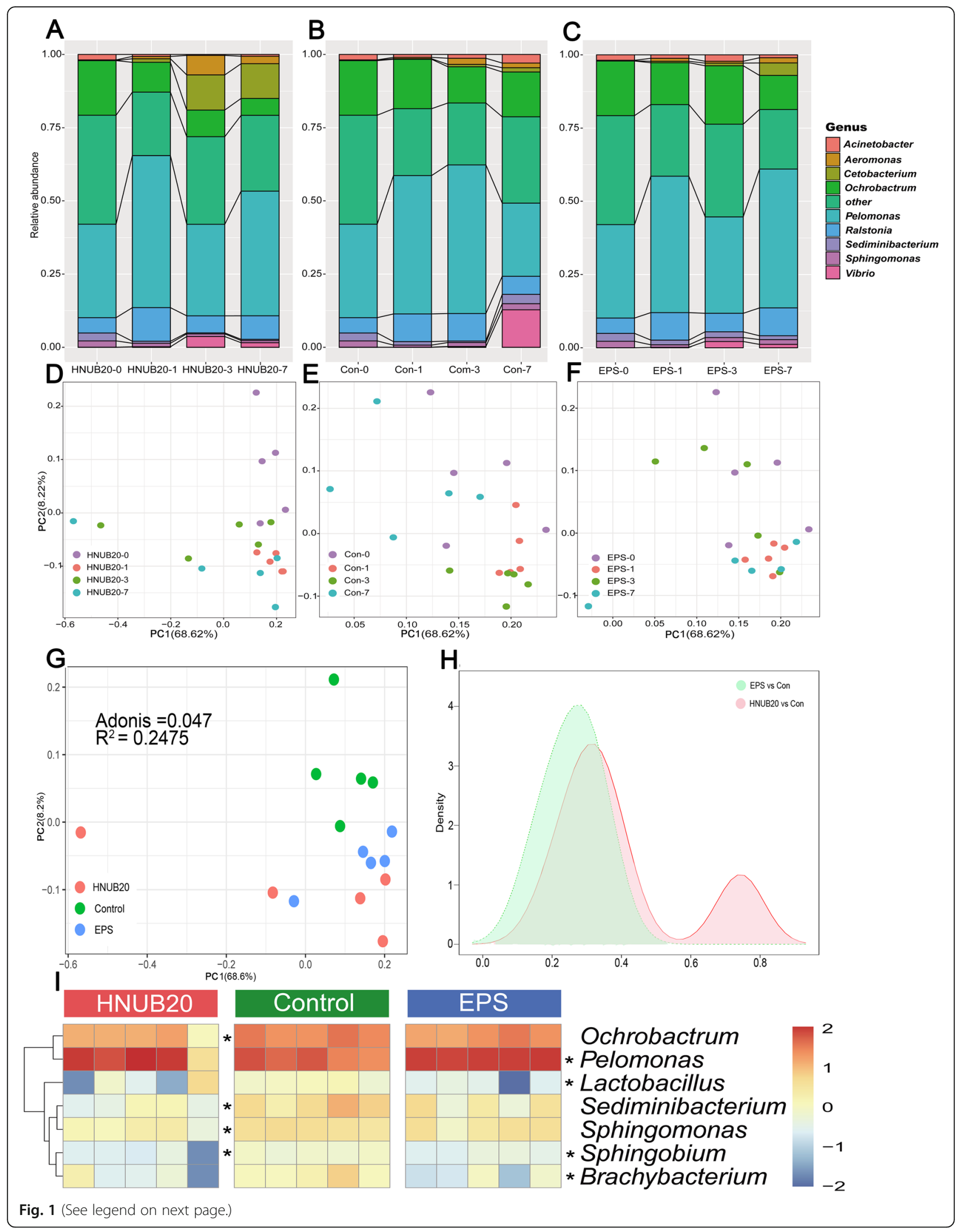


(See figure on previous page.)

Fig. 1 Bacterial community composition and comparison of different genera. The histogram shows changes in the core microbiota at different time points (a-c). Principal coordinate analysis (PCoA) reveals the microbial structure based on weighted UniFrac distances in the healthy zebrafish at different time points (d-f), seventh days of PCOA could be found in (g). The density plot shows the degree of change after EPS and Lactobacillus fermentum HNUB20 treatment (Fig. H1). The different genera are illustrated with a heatmap, and the star represents different genera compared with the control group (i) using the Wilcoxon rank sum test, $P<0.01$ )

metabolites were involved in the bioactivity performance of EPS and HNUB20. This may be one of the potential mechanisms of EPS and HNUB20 regulation of immune immunity.

\section{Discussion}

In this study, we found EPSs and HNUB20 can shake the structure of intestinal microbiota, while the effects on specific genera were obviously different. The specific manifestation is that there is no same differential genus. Although the secretion of EPS is a physiological characteristic of HNUB20, from its point of view of intestinal microbiota, we should evaluate a strain more comprehensively, rather than just one characteristic. Then, commonalities between EPS and HNUB20 were found that is not strong enough to stimulate SCFAs and immunity. We think that the reason is that the regulation of specific intestinal microbes, including SCFAs-secreting bacteria is still insufficient.

Gut microbiota composition may be important an indicator of host health $[24,25]$. In our study, no significant differences in alpha diversity were found among the three groups of healthy zebrafish. An earlier study showed a sex dependent effect on alpha diversity, whereby alpha diversity was significantly different among males, with no difference among females [26]. Thus, sex may affect changes in alpha diversity. Our research included females and males that were not evaluated separately. It has been reported that EPS from Lactobacillus buchneri TCP016 had no effect on mouse alpha diversity of microbiota [27]. In general, larger sample sizes may be needed to confirm the factors affecting alpha diversity. Regardless, the core microbiota showed changes after EPS and L. fermentum HNUB20 treatment. Nine genera were defined as the core microbiota with average relative abundances greater than 1\%. Three genera, Aeromonas, Vibrio and Cetobacterium, are considered to belong to core intestinal microbiota in domesticated and caught zebrafish [28]. Vibrio and Cetobacterium are also known as core microbiota of adult zebrafish [29], which is consistent with our study. L. fermentum HNUB20 inhibited the growth of pathogenic bacteria (Ochrobactrum) [30, 31], and bacteria associated with disease biomarkers (Sediminibacterium, Sphingomonas, Sphingobium) [32-34]. HNUB20 derived EPS can decrease the level of Pelomonas, which is a biomarker of bladder cancer [32]. It also inhibited the growth of Brachybacterium, which is an end-stage renal disease biomarker (Brachybacterium) [35].

Most related studies to date have been performed in vitro. However, additional in vivo research is needed to evaluate the health benefits and properties of EPSs and EPS-producing strains. One of the first questions is

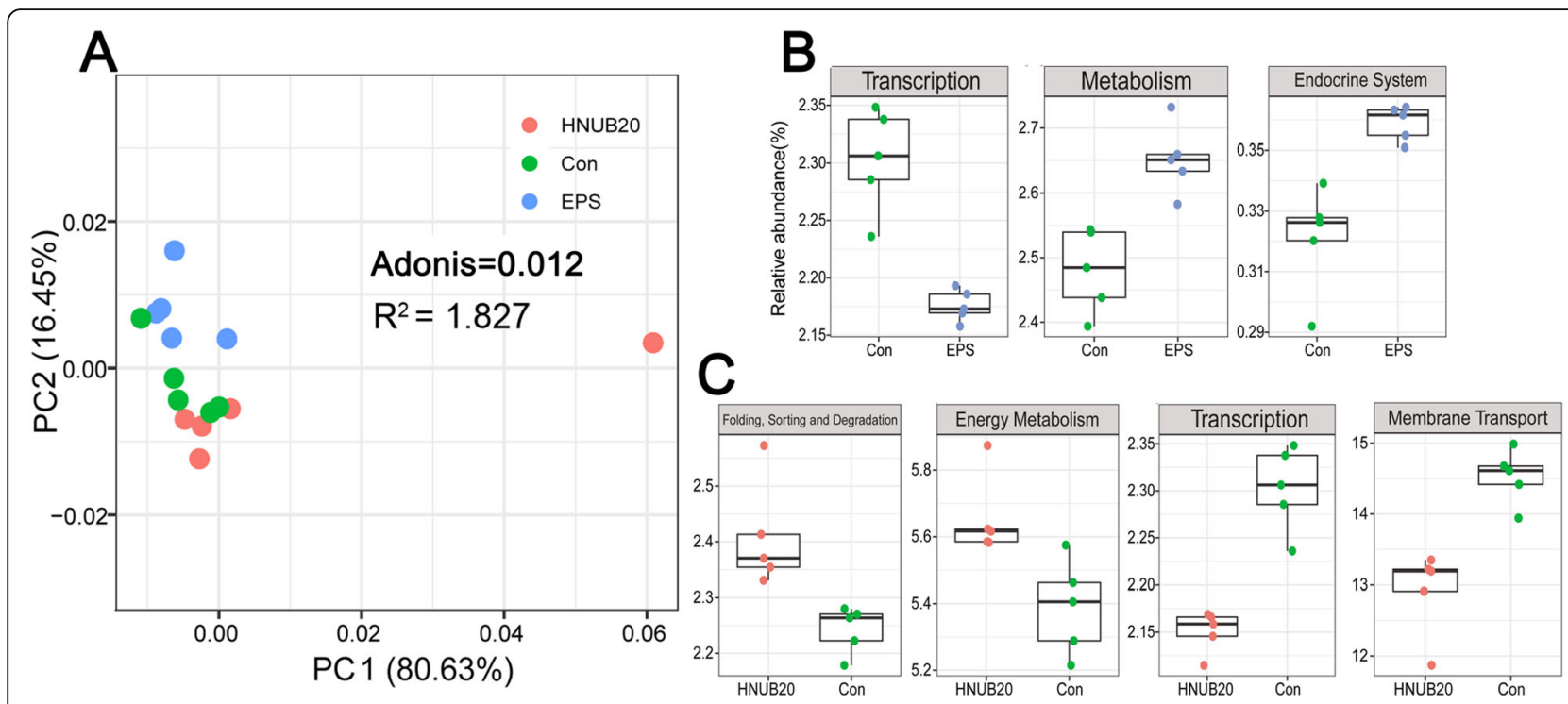

Fig. 2 Principal component analysis (PCA) of metabolic pathways and different metabolic pathways in level 2. Adonis analysis was conducted to permute $P$ value by 999 permutations (a). The Wilcoxon rank sum test was used and considered significant at $P<0.01$ (b) 


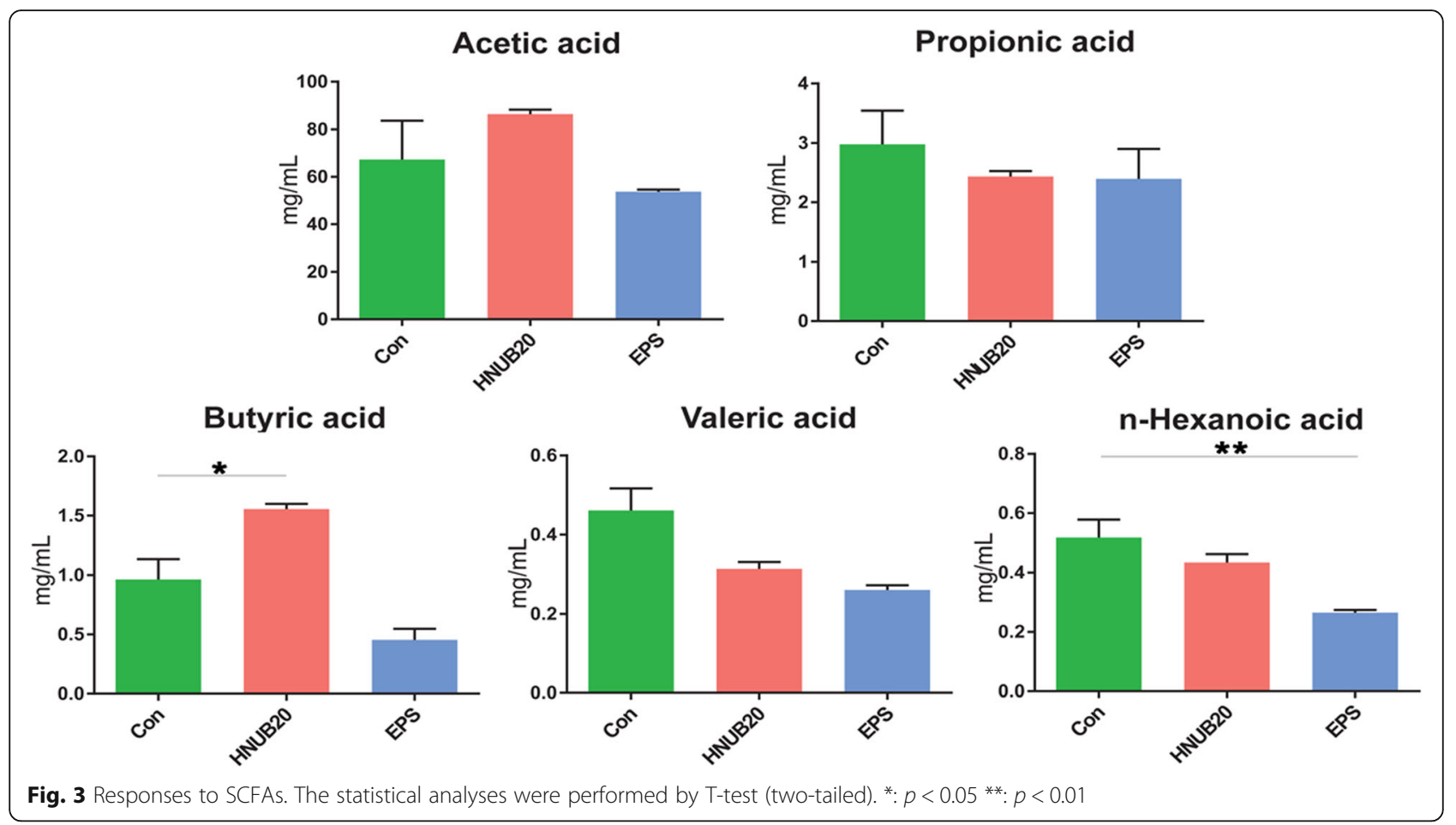

whether EPSs contribute to the colonization of strains. We detected the abundance of Lactobacillus fermentum by qPCR based on specific primers of Lactobacillus fermentum [36], and the results can be found in more detail (see Additional file 2). However, no significant differences were found. In fact, adhesion is one of the properties frequently assessed in the search for potential probiotic strains [37]. Several studies have been performed with Caco-2 cells in vitro, showing that EPS can help other bacteria strains to adhere to these cells [20]. On the other hand, another study showed that non-EPSproducing strains persist longer in mice [38] and that the presence of EPS surrounding the bacterial surface might reduce adherence [39]. Nonetheless, strain adherence to human GI cells in vitro may be a poor indicator of in vivo colonization due to a myriad of host and microbiome factors that are absent in the in vitro setting [40]. In addition, EPS may not be a good complete carbon source for Lactobacillus. Most studies have reported increases in Bifidobacterium abundance by EPS treatment $[41,42]$. EPS may have two sides (promote or inhibit colonization) for EPS-producing Lactobacillus to the host and the microbiota.

The SCFAs were considered as the key bacterial metabolites, which can modulate immune response in gut by controlling the expression of genes involved in
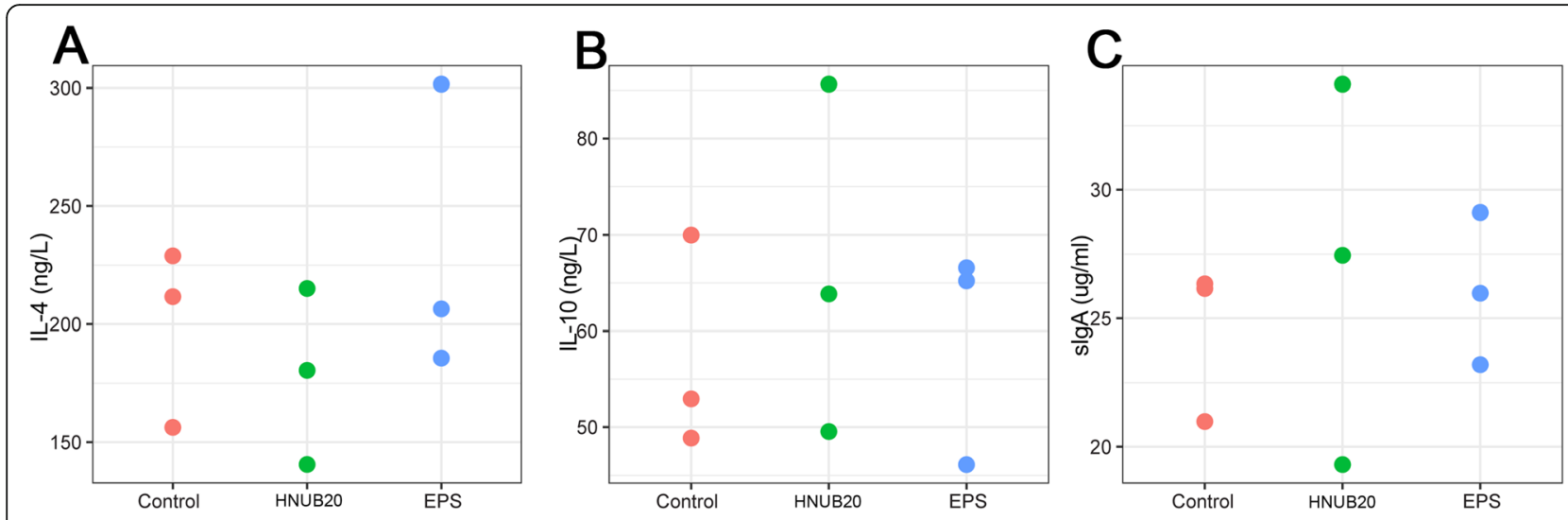

Fig. 4 Responses to immune indexes. The statistical analyses were performed by T-test (two-tailed). *: $p<0.05^{* *}: p<0.01$ 


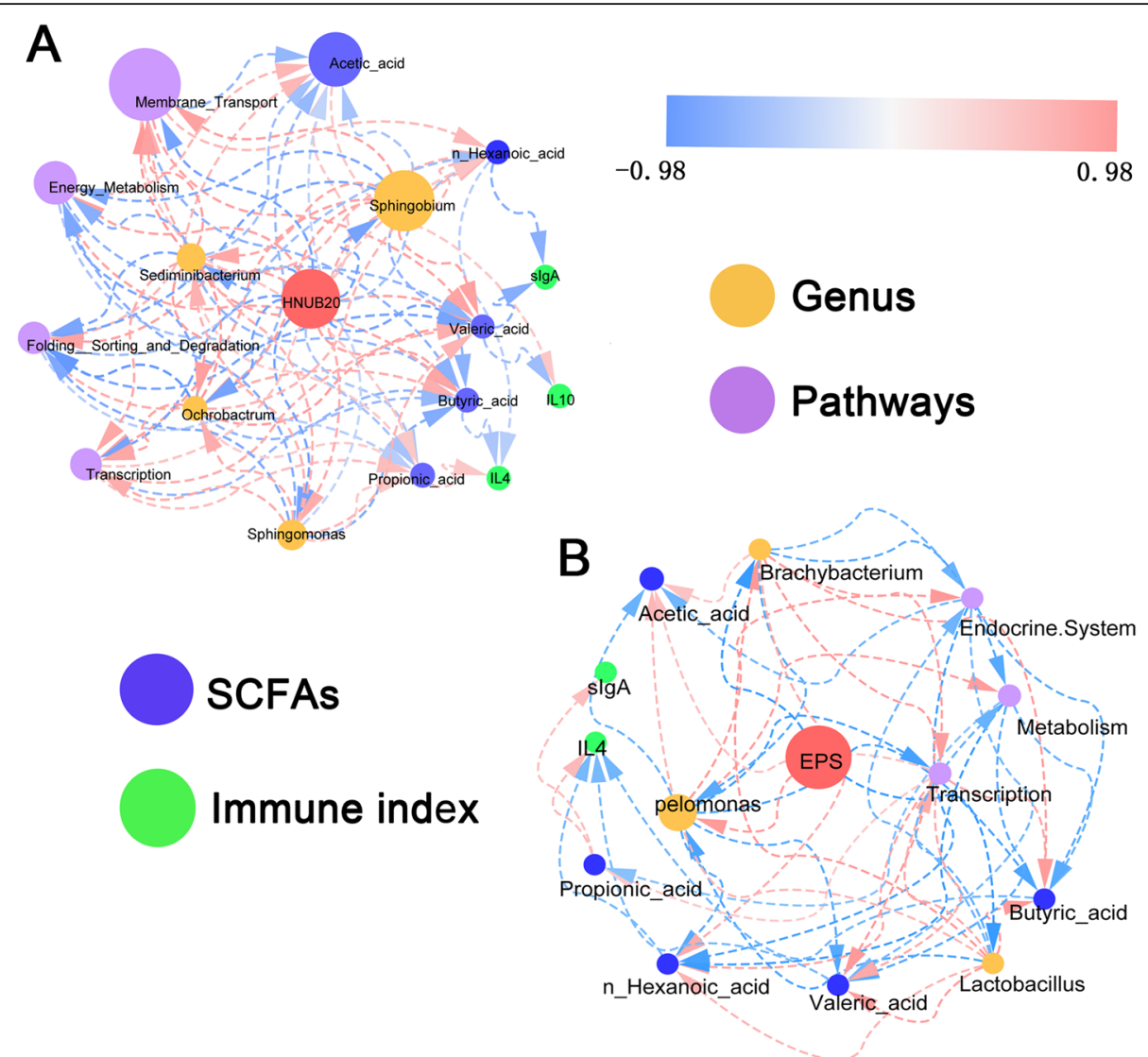

Fig. 5 Correlation network analysis among EPS, Lactobacillus fermentum HNUB20, different genera, metabolic pathways, SCFAs and immune indexes. Correlations were estimated by Spearman rank correlation coefficient. Absolute correlations below 0.4 were masked to show all exclusively significant signals. The colour (red, positive; blue, negative) are proportional to the correlations. The node size of genera and pathways is proportional to the mean abundance in the respective cohorts

synthesis of molecules necessary for plasma B cell differentiation [43]. A study by Rio-Covian et al. revealed that EPS promotes the release of SCFAs [44], while limited effect also was found with EPS treatment [45]. Propionic acid and butyric acid contribute to an anti-inflammatory by induce apoptosis of neutrophils [46]. Butyric acid also exerts immunomodulatory effect by effecting on intestinal macrophages result in a reduction in the production of pro-inflammatory cytokines [47]. However, based on our data, no consistent responses were found on SCFAs with EPS and HNUB20 treatment in the healthy zebrafish, though HNUB20 can enhance intestinal immune function and promote intestinal health through butyrate production. IgA is the most abundant immunoglobulin subtype present in epithelial mucus, which captures antigens and prevents them from binding to cell surface receptors. Dendritic cells (DCs) operate on mesenteric lymph nodes by sampling of polysaccharide and bacteria, which is responsible for regulatory $\mathrm{T}$ (Treg) to produce IL-10, and $\operatorname{IgA}^{+} \mathrm{B}$ cell (responsible for $\operatorname{IgA}^{+}$ plasma cell to produce IgA) [48]. EPS extracted from $L$. mesenteroides strain NTM048 (ranging in size from 10 to $40 \mathrm{kDa}$ ) possesses the ability to increase IgA levels [49]. In addition, EPS from Lactobacillus casei WXD030 (average molecular weight of $37,370 \mathrm{Da}$ ) promoted IL-4 and IL-10 expression [50]. However, a limited response to EPS of strain HNUB20 (EPS was estimated to be 45, $065 \mathrm{Da}$ ) was found in this study in the healthy zebrafish. Some previous studies have focused on large or high molecular weight EPSs, which appear to act as suppressors of the immune response, which may explain the difference in responses observed [51] in the healthy zebrafish. Collectively, we speculated that the HNUB20 may act as a transient microbe that does not permanently colonize the gut yet still shake the composition of the host intestinal microbiota and have a limited impact on SCFAs presence and intestinal inflammation of the host.

\section{Conclusions}

In conclusion, the present study described the dynamic profile of the response of the zebrafish intestinal microbiota, SCFAs and immune indexes to the HNUB20 and EPS intakes. The present intestinal microbial model- 
based study indicated that EPSs and high-yield EPSproducing Lactobacillus can shake the structure of intestinal microbiota, but cannot change SCFAs presence and intestinal inflammation.

\section{Methods}

\section{Screening of EPS-producing lactic acid bacteria and} preparation of crude EPS

In our previous studies, we isolated and identified 178 lactic acid bacteria from fermented seafoods of the Hainan area, China [52]. These lactic acid bacteria were screened for EPS production. The methods of Ai et al. [53] were adopted and improved. Cultures were inoculated at $2 \%(\mathrm{v} / \mathrm{v})$ and cultured in MRS medium for approximately $48 \mathrm{~h}$ at $37^{\circ} \mathrm{C}$. Trichloroacetic acid (TCA) with a final concentration of $3 \%$ was added, and then placed in the refrigerator for 4 hours to precipitate proteins [54]. The clear supernatant was collected after centrifugation at $12,000 \mathrm{~g}$ for $15 \mathrm{~min}$, and EPS was precipitated by adding three volumes of cold ethanol followed by overnight storage at $4{ }^{\circ} \mathrm{C}$. After centrifugation $(12,000 \times \mathrm{g}$ for $20 \mathrm{~min})$, EPS was resuspended in $\mathrm{dd}_{2} \mathrm{O}$, dialyzed against running water for $48 \mathrm{~h}(8000-$ $14,000 \mathrm{Da}$ ), and collected by centrifugation at $12,000 \times \mathrm{g}$ for $30 \mathrm{~min}$. Pelleted EPS was resuspended in $\mathrm{ddH}_{2} \mathrm{O}$. To determine the yield of EPS, the phenol-sulfuric acid method was used with glucose as a standard [55] $(\mathrm{y}=$ 0.0179x-0.0302 $\left.R^{2}=0.9967\right)$. Lactobacillus fermentum HNUB20 was found to produce the most EPS, at approximately $98.8 \mathrm{mg} / \mathrm{L}$ (the screening results can be found in supplemental material [see Additional file 5]). The crude EPS of strain HNUB20 was prepared from 10 $\mathrm{L}$ culture by ethanol precipitation, followed by dialysis and freeze drying. Isolated EPS was stored at $4{ }^{\circ} \mathrm{C}$ for further experiments. The HPLC analysis of EPS is presented (see Additional file 1). Based on the results, the molecular weight of EPS is 45,065 Da.

\section{Animal experimental design, diet preparation and sample collection}

Adult zebrafish (including male and female adults, 3 months old, average mass $500 \pm 50 \mathrm{mg}$, average body length $3.2 \pm 0.1 \mathrm{~cm}$ ) used in the experiment were obtained from a local supplier in Haikou and cultured at the aquatic experimental animal facility of the College of Food Science and Technology, Hainan University. The zebrafish were acclimated to laboratory conditions for 14 days in aerated dechlorinated tap water prior to experiments. According to the standard zebrafish breeding protocol, the acclimation and subsequent treatment period were conducted at $28 \pm 0.5^{\circ} \mathrm{C}$, with a light/dark cycle of $14 \mathrm{~h} / 10 \mathrm{~h}$. The water was replaced at 9 a.m. every day to avoid the growth of pathogenic bacteria. The fish were fed commercial feed (Sanyou Chuangmei,
China) daily in the amount of $3 \%$ of the total zebrafish weight. After 14 days of acclimation, zebrafish were randomly distributed into three groups (Control, EPS and HNUB20 Group), with three 3-L tanks for each group $(24 \mathrm{~cm} \times 13 \mathrm{~cm} \times 12 \mathrm{~cm})$ and 16 fish in each tank. The EPS group zebrafish were fed the control diet plus $1 \%$ $(\mathrm{m} / \mathrm{m})$ crude EPS. L. fermentum HNUB20 cells were pelleted by centrifugation and resuspended in $50 \mu \mathrm{L}$ of saline, which was mixed with feed at $8.0 \log _{10} \mathrm{CFU} / \mathrm{g}$ diet.

Zebrafish were anesthetized by immersion in $0.2 \mathrm{mg} / \mathrm{ml}$ tricaine (MS-222) [56] and then placed on an ice plate for dissection sampling. All experiments were carried out in accordance with the National Institutes of Health Guide for the Care and Use of Laboratory Animals (NIH Publications No.8023). All of the experimental and animal care procedures were approved by the Ethics Committee of Hainan University (Permit Number: HNU-EC-20180701). Fifteen intestinal samples of the healthy zebrafish were collected at four points during the experiment (baseline, first day, third days, and seventh days). The whole intestines (esophagus to anus) from three animals were mixed into one sample, giving a totally five pooled samples for further microbiota analysis. The flow chart can be found in more detail [see Additional file 3].

\section{DNA extraction}

$500 \mu \mathrm{L}$ of TE (10 Tris-EDTA, pH 8.0 Tris- $\mathrm{HCl} 0.1 \mathrm{~mol} /$ $\mathrm{L})$ was added to homogenize the samples. Samples were stored at $-20^{\circ} \mathrm{C}$ until analysis. Metagenomic DNA was extracted from the zebrafish intestinal samples using a CWBIO Stool Genomic DNA Kit (CW2092, CWBIO, China). We assessed the quality of the metagenomic DNA by $0.8 \%$ agarose gel electrophoresis before sequencing. Isolated metagenomic DNA was stored at $-20^{\circ} \mathrm{C}$ and used as the template for further analysis.

\section{DNA sequencing}

The Shanghai Personal Biotechnology company amplified the DNA coding for the V3-V4 region of the $16 \mathrm{~S}$ ribosomal RNA (rRNA) gene, as described previously [57]. We added a set of 6-nucleotide barcodes to the universal forward primer 338F (5'-ACTCCTACGG GAGGCAGCA-3') and reverse primer 806R (5'-GGAC TACHVGGGTWTCTAAT-3'). Quantification of PCR products was performed using an Agilent DNA 1000 Kit and Agilent 2100 Bioanalyzer (Agilent Technologies, USA) according to the manufacturer's instructions. The amplification products were pooled in equimolar ratios at a final concentration of $100 \mathrm{nmol} / \mathrm{L}$ each and sequenced using the Illumina MiSeq platform with the barcoded primers. 


\section{SCFA analysis}

Short fatty acids were analyzed by a gas chromatographymass spectrometry (GC-MS) as previously described [58]. In short, the whole gut tissue samples (esophagus to anus) of the healthy zebrafish from five animals were weighted, followed by addition of $2 \mathrm{~mL} \mathrm{H}_{2} \mathrm{SO}_{4}(0.5 \mathrm{~mol} / \mathrm{L})$ and extracted $30 \mathrm{~min}$ by ultrasonic vibration $\left(40{ }^{\circ} \mathrm{C}, 35 \mathrm{kHz}\right)$. Then, $1 \mathrm{~mL}$ of diethyl ether was added, samples were stored at $4{ }^{\circ} \mathrm{C}$ for $5 \mathrm{~min}$ and centrifuged $(12,000 \mathrm{~g}$ for 5 min). Finally, $100 \mu \mathrm{L}$ of acetone was added for GC-MS after diethyl ether was removed. An Agilent 7890A-5975C GC-MS and Agilent DB-WAX $(0.25 \mathrm{~mm} \times 0.25 \mu \mathrm{m} \times 50$ $\mathrm{m})$ columns were used. The GC conditions were as follows: inlet temperature: $250{ }^{\circ} \mathrm{C}$; carrier gas flow rate: 1.5 $\mathrm{mL} / \mathrm{min}$; shunt ratio 3:1 and injection amount of sample was $1 \mu \mathrm{L}$. The GC temperature program was as follows: $70{ }^{\circ} \mathrm{C}$ hold for $3.0 \mathrm{~min}$, increase to $200^{\circ} \mathrm{C}$ by $10^{\circ} \mathrm{C} / \mathrm{min}$, $200^{\circ} \mathrm{C}$ hold for $2.0 \mathrm{~min}$, increased to $180^{\circ} \mathrm{C}$ by $8^{\circ} \mathrm{C} / \mathrm{min}$, $180^{\circ} \mathrm{C}$ hold for $10.0 \mathrm{~min}$, and increased to $250{ }^{\circ} \mathrm{C}$ for 10 min by $15^{\circ} \mathrm{C} / \mathrm{min}$. The MS conditions were as follows: the ion source chamber was set at $230^{\circ} \mathrm{C}$ with the transfer line temperature set to $250^{\circ} \mathrm{C}$, the electron energy was $70 \mathrm{eV}$, and the full scan was 35 to $550 \mathrm{Da}$. All samples were tested three times and the average value was used as the result.

\section{Enzyme-linked immunosorbent assays (ELISA)}

The whole gut tissue (esophagus to anus) of all group from two zebrafish were mixed into one sample, weighed, homogenized in $7.2 \mu \mathrm{L} / \mathrm{mg}$ PBS (pH 6.0, 50 $\mathrm{mM}$ ) and centrifuged (13,000 g for $5 \mathrm{~min}$ ), giving totally three pooled samples for further analysis, all samples were tested three times and the average value was used as the result. Supernatants were used for interleukin-4 (IL-4), interleukin-10 (IL-10), and secretory IgA (sIgA) quantification using ELISA kits, described in the manual instructions (X-Y Biotechnology, Shanghai, CN). Absorption at $450 \mathrm{~nm}$ was determined using a microplate reader (SpectraMax M2, MD).

\section{Bioinformatics and statistical analyses}

Low-quality sequences from $16 \mathrm{~S}$ rDNA sequencing results were removed after trimming based on the original raw data. FLASH and QIIME (v1.8.0) USEARCH software were used for further sequence quality analysis [59]. Representative OTUs (operational taxonomic unit) were selected and annotated using the Greengenes database [60]. PICRUSt (Phylogenetic Communities by Reconstruction of Unobserved States) was applied to predict the functional features of the zebrafish intestinal microbiota based on the OTU table [61]. Statistical analyses were conducted using $\mathrm{R}$ software. The data were corrected for multiple testing, and the $p$-value was the adjusted p-value by the "p.adjust" command in R.
Differences in the abundance of genera and metabolic pathways were assessed by Wilcoxon rank sum test and considered significant at $P<0.01$. Only those pathways and genera with more than $0.1 \%$ average abundance and only genera present in at least $20 \%$ of the samples were used for statistical analysis by Wilcoxon rank sum test. Graph drawing and Principal component analysis (PCA) were performed using the ggplot2 package [62]. Principal coordinate analysis (PCoA) of weighted Unifrac distance was performed in $\mathrm{R}$ using the ade4 package [63]. Adonis analysis was conducted using the vegan package, and the permuted $P$ value was obtained by 999 permutations. Heatmaps, which were employed to show the presence of different bacterial genera were generated using the pheatmap package. Correlations between microbes among groups were calculated using the Spearman rank correlation (psych package) coefficient and visualized as a network in Cytoscape (v 3.4) [64]. T-test (two-tailed) was used to compare the difference for cytokines and SCFAs.

\section{Supplementary information}

Supplementary information accompanies this paper at https://doi.org/10. 1186/s12866-020-01990-6.

Additional file 1. The HPLC analysis of EPS.

Additional file $\mathbf{2}$ The $\mathrm{qPCR}$ results of Lactobacillus fermentum.

Additional file 3 . The workflow of the study.

Additional file 4. The relative abundance of citrate cycle (TCA cycle) among the three groups.

Additional file $\mathbf{5}$. The screening results.

Additional file 6. The abundance of bacteria at phyla level.

Additional file 7. The abundance of core genera.

Additional file 8. The abundance of metabolic pathways in level 2.

\section{Abbreviations}

EPSs: Exopolysaccharides; LAB: Lactic acid bacteria; PCoA: Principal coordinate analysis; PICRUSt: Phylogenetic Communities by Reconstruction of Unobserved States

Acknowledgements

Not applicable.

Authors' contributions

XZ and JZ designed the study. SJ, CM and HG collected the samples. HG and $\mathrm{DH}$ processed and sequenced the samples. $\mathrm{HC}, \mathrm{SH}$ and $\mathrm{CM}$ analyzed the data. JZ, HC, XZ and CM wrote the paper with contributions and final approval from all of the authors.

\section{Funding}

This research was funded by Key $R$ \& D programs in Hainan, grant number ZDYF2019150 and ZDYF2018111; the Scientific Research Foundation of Hainan University, grant number KYQD (ZR)1713. The funding body has no role in study. design, collection, analysis, data interpretation and in writing the manuscript.

Availability of data and materials

The datasets generated during the current study are available in the [NCBI] repository, [https://www.ncbi.nlm.nih.gov/bioproject/PRJNA530096]. 


\section{Ethics approval and consent to participate}

All experiments were carried out in accordance with the National Institutes of Health Guide for the Care and Use of Laboratory Animals ( $\mathrm{NIH}$ Publications No.8023). All of the experimental and animal care procedures were approved by the Ethics Committee of Hainan University (Permit Number: HNU-EC-20180701)

\section{Consent for publication}

Not applicable.

\section{Competing interests}

The authors declare that they have no competing interests.

\section{Author details}

${ }^{1}$ College of Food Science and Engineering, School of Life and Pharmaceutical Sciences, Hainan University, Haikou 570228, Hainan, P. R. China. ${ }^{2}$ Key Laboratory of Tropical Biological Resources, Ministry of Education, Hainan University, Haikou 570228, Hainan, P. R. China. ${ }^{3}$ Single-Cell Center, Qingdao Institute of Bioenergy and Bioprocess Technology, Chinese Academy of Sciences, Qingdao 266101, Shandong, People's Republic of China.

Received: 24 May 2020 Accepted: 30 September 2020 Published online: 06 October 2020

\section{References}

1. Shao Y, Huo D, Peng Q, Pan Y, Jiang S, Liu B, et al. Lactobacillus plantarum HNU082-derived improvements in the intestinal microbiome prevent the development of hyperlipidaemia. Food Funct. 2017;8(12):4508-16. https://doi.org/10.1039/c7fo00902j.

2. Zhang J, Sun Z, Jiang S, Bai X, Ma C, Peng Q, et al. Probiotic Bifidobacterium lactis $\vee 9$ regulates the secretion of sex hormones in polycystic ovary syndrome patients through the gut-brain Axis. mSystems. mSystems. 2019; 4(2):e00017. https://doi.org/10.1128/mSystems.00017-19.

3. Hidalgo-Cantabrana C, Sanchez B, Milani C, Ventura M, Margolles A, RuasMadiedo P. Genomic overview and biological functions of exopolysaccharide biosynthesis in Bifidobacterium spp. Appl Environ Microbiol. 2014;80(1):9-18.

4. Jiang $Y Y$, Yang ZN. A functional and genetic overview of exopolysaccharides produced by lactobacillus plantarum. J Funct Foods. 2018;47:229-40.

5. Korcz E, Kerenyi Z, Varga L. Dietary fibers, prebiotics, and exopolysaccharides produced by lactic acid bacteria: potential health benefits with special regard to cholesterol-lowering effects. Food Funct. 2018;9(6):3057-68. https://doi.org/10.1039/c8fo00118a.

6. Ndeh D, Gilbert HJ. Biochemistry of complex glycan depolymerisation by the human gut microbiota. FEMS Microbiol Rev. 2018;42(2):146-64.

7. Salazar N, Ruas-Madiedo P, Kolida S, Collins M, Rastall R, Gibson G, et al. Exopolysaccharides produced by Bifidobacterium longum IPLA E44 and Bifidobacterium animalis subsp. lactis IPLA R1 modify the composition and metabolic activity of human faecal microbiota in $\mathrm{pH}$-controlled batch cultures. Int J Food Microbiol. 2009;135(3):260-7. https://doi.org/10.1016/j. ijfoodmicro.2009.08.017.

8. Zhang J, Zhao X, Jiang Y, Zhao W, Guo T, Cao Y, et al. Antioxidant status and gut microbiota change in an aging mouse model as influenced by exopolysaccharide produced by lactobacillus plantarum YW11 isolated from Tibetan kefir. J Dairy Sci. 2017;100(8):6025-41. https://doi.org/10.3168/jds. 2016-12480

9. Kim M, Qie Y, Park J, Kim CH. Gut microbial metabolites fuel host antibody responses. Cell Host Microbe. 2016;20(2):202-14. https://doi.org/10.1016/j. chom.2016.07.001.

10. Zhou X, Qi W, Hong T, Xiong T, Gong D, Xie M, et al. Exopolysaccharides from lactobacillus plantarum NCU116 regulate intestinal barrier function via STAT3 signaling pathway. J Agric Food Chem. 2018;66(37):9719-27. https://doi.org/10.1021/acs.jafc.8b03340.

11. Dinic M, Pecikoza U, Djokic J, Stepanovic-Petrovic R, Milenkovic M, Stevanovic M, et al. Exopolysaccharide produced by probiotic strain lactobacillus paraplantarum BGCG11 reduces inflammatory Hyperalgesia in rats. Front Pharmacol. 2018;9:1. https://doi.org/10.3389/fphar.2018.00001.
12. Lim J, Kale M, Kim DH, Kim HS, Chon JW, Seo KH, et al. Antiobesity effect of exopolysaccharides isolated from kefir grains. J Agric Food Chem. 2017. 65(46):10011-9. https://doi.org/10.1021/acs.jafc.7b03764.

13. Russo P, Lopez P, Capozzi V, de Palencia PF, Duenas MT, Spano G, et al. Beta-glucans improve growth, viability and colonization of probiotic microorganisms. Int J Mol Sci. 2012;13(5):6026-39. https://doi.org/10.3390/ ijms13056026.

14. Rico CW, Shin JH, Um IC, Kang MY. Cholesterol-lowering action and Antioxidative effects of microbial gum in C57BL/6N mice fed a high fat diet. Biotechnol Bioproc E. 2011;16(1):167-72.

15. Bello FD, Walter J, Hertel C, Hammes WP. In vitro study of prebiotic properties of Levan-type exopolysaccharides from lactobacilli and nondigestible carbohydrates using denaturing gradient gel electrophoresis. Syst Appl Microbiol. 2001;24(2):232-7. https://doi.org/10.1078/0723-2020-00033.

16. Gaulke CA, Barton CL, Proffitt S, Tanguay RL, Sharpton TJ. Triclosan exposure is associated with rapid restructuring of the microbiome in adult Zebrafish. PLoS One. 2016;11(5):e0154632. https://doi.org/10.1371/journal.pone.0154632.

17. Jemielita M, Taormina MJ, Burns AR, Hampton JS, Rolig AS, Guillemin K, et al. Spatial and temporal features of the growth of a bacterial species colonizing the zebrafish gut. MBio. 2014;5(6):e01751. https://doi.org/10.1128/ mBio.01751-14.

18. Stephens WZ, Wiles TJ, Martinez ES, Jemielita M, Burns AR, Parthasarathy R, et al. Identification of population bottlenecks and colonization factors during assembly of bacterial communities within the Zebrafish intestine. MBio. 2015;6(6):e01163-15. https://doi.org/10.1128/mBio.01163-15.

19. Chuang LS, Morrison J, Hsu NY, Labrias PR, Nayar S, Chen E, et al. Zebrafish modeling of intestinal injury, bacterial exposures and medications defines epithelial in vivo responses relevant to human inflammatory bowel disease. Dis Model Mech. 2019;12(8):dmm037432. https://doi.org/10.1242/dmm. 037432.

20. Perez-Ramos A, Mohedano ML, Pardo MA, Lopez P. Beta-Glucan-producing Pediococcus parvulus 2.6: test of probiotic and Immunomodulatory properties in Zebrafish models. Front Microbiol. 2018;9:1684. https://doi.org/ 10.3389/fmicb.2018.01684

21. Davis DJ, Doerr HM, Grzelak AK, Busi SB, Jasarevic E, Ericsson AC, et al. Lactobacillus plantarum attenuates anxiety-related behavior and protects against stress-induced dysbiosis in adult zebrafish. Sci Rep. 2016;6:33726. https://doi.org/10.1038/srep33726.

22. Astudillo-Garcia C, Bell JJ, Webster NS, Glasl B, Jompa J, Montoya JM, et al. Evaluating the core microbiota in complex communities: a systematic investigation. Environ Microbiol. 2017;19(4):1450-62. https://doi.org/10.1111/ 1462-2920.13647.

23. Gong X, Jiang S, Tian H, Xiang D, Zhang J. Polyphenols in the fermentation liquid of Dendrobium candidum relieve intestinal inflammation in Zebrafish through the intestinal microbiome-mediated immune response. Front Immunol. 2020;11:1542. https://doi.org/10.3389/fimmu.2020.01542.

24. Guo Z, Zhang J, Wang Z, Ang KY, Huang S, Hou Q, et al. Intestinal microbiota distinguish gout patients from healthy humans. Sci Rep. 2016;6: 20602. https://doi.org/10.1038/srep20602.

25. Qin N, Yang F, Li A, Prifti E, Chen Y, Shao L, et al. Alterations of the human gut microbiome in liver cirrhosis. Nature. 2014;513(7516):59-64. https://doi. org/10.1038/nature13568.

26. Ma YB, Song LY, Lei $Y$, Jia PP, Lu CJ, Wu JF, et al. Sex dependent effects of silver nanoparticles on the zebrafish gut microbiota. Environ Sci-Nano. 2018; 5(3):740-51.

27. Xu R. Aruhan, Xiu L, sheng S, Liang Y, Zhang H, et al. exopolysaccharides from lactobacillus buchneri TCP016 attenuate LPS- and d-GalN-induced liver injury by modulating the gut microbiota. J Agric Food Chem. 2019;67(42): 11627-37. https://doi.org/10.1021/acs.jafc.9b04323.

28. Roeselers G, Mittge EK, Stephens WZ, Parichy DM, Cavanaugh CM, Guillemin K, et al. Evidence for a core gut microbiota in the zebrafish. Isme J. 2011; 5(10):1595-608

29. Stephens WZ, Burns AR, Stagaman K, Wong S, Rawls JF, Guillemin K, et al. The composition of the zebrafish intestinal microbial community varies across development. Isme J. 2016;10(3):644-54.

30. Han F, Wang X, Guo J, Qi C, Xu C, Luo Y, et al. Effects of glycinin and betaconglycinin on growth performance and intestinal health in juvenile Chinese mitten crabs (Eriocheir sinensis). Fish Shellfish Immunol. 2019;84: 269-79. https://doi.org/10.1016/j.fsi.2018.10.013.

31. Walujkar SA, Kumbhare SV, Marathe NP, Patangia DV, Lawate PS, Bharadwaj $\mathrm{RS}$, et al. Molecular profiling of mucosal tissue associated microbiota in 
patients manifesting acute exacerbations and remission stage of ulcerative colitis. World J Microbiol Biotechnol. 2018;34(6):76. https://doi.org/10.1007/ s11274-018-2449-0.

32. Liu F, Liu A, Lu X, Zhang Z, Xue $Y$, Xu J, et al. Dysbiosis signatures of the microbial profile in tissue from bladder cancer. Cancer Med. 2019;8(16):6904. https://doi.org/10.1002/cam4.2419.

33. Mei QX, Huang CL, Luo SZ, Zhang XM, Zeng Y, Lu YY. Characterization of the duodenal bacterial microbiota in patients with pancreatic head cancer vs. healthy controls. Pancreatology. 2018;18(4):438-45. https://doi.org/10. 1016/j.pan.2018.03.005.

34. Qiu J, Zhou H, Jing Y, Dong C. Association between blood microbiome and type 2 diabetes mellitus: a nested case-control study. J Clin Lab Anal. 2019; 33(4):e22842. https://doi.org/10.1002/jcla.22842.

35. Vaziri ND, Wong J, Pahl M, Piceno YM, Yuan J, DeSantis TZ, et al. Chronic kidney disease alters intestinal microbial flora. Kidney Int. 2013;83(2):308-15. https://doi.org/10.1038/ki.2012.345.

36. Matsuda K, Tsuji H, Asahara T, Matsumoto K, Takada T, Nomoto K. Establishment of an analytical system for the human fecal microbiota, based on reverse transcription-quantitative PCR targeting of multicopy rRNA molecules. Appl Environ Microbiol. 2009;75(7):1961-9. https://doi.org/10. 1128/AEM.01843-08

37. Guo XZ, Li J, Ran C, Wang AR, Xie MX, Xie YD, et al. Dietary nucleotides can directly stimulate the immunity of zebrafish independent of the intestinal microbiota. Fish Shellfish Immun. 2019;86:1064-71.

38. Denou E, Pridmore RD, Berger B, Panoff JM, Arigoni F, Brussow H. Identification of genes associated with the long-gut-persistence phenotype of the probiotic lactobacillus johnsonii strain NCC533 using a combination of genomics and transcriptome analysis. J Bacteriol. 2008;190(9):3161-8. https://doi.org/10.1128/JB.01637-07.

39. Castro-Bravo N, Wells JM, Margolles A, Ruas-Madiedo P. Interactions of surface exopolysaccharides from Bifidobacterium and lactobacillus within the intestinal environment. Front Microbiol. 2018;9:2426. https://doi.org/10. 3389/fmicb.2018.02426.

40. Suez J, Zmora N, Segal E, Elinav E. The pros, cons, and many unknowns of probiotics. Nat Med. 2019;25(5):716-29. https://doi.org/10.1038/s41591-0190439-x.

41. Hongpattarakere T, Cherntong N, Wichienchot S, Kolida S, Rastall RA. In vitro prebiotic evaluation of exopolysaccharides produced by marine isolated lactic acid bacteria. Carbohydr Polym. 2012;87(1):846-52.

42. Hung TV, Wanatanbe J, Yonejima Y, Hisa K, Yamamoto Y, Suzuki T. Exopolysaccharides from Leuconostoc mesenteroides attenuate chronic kidney disease in mice by protecting the intestinal barrier. J Funct Foods. 2019;52:276-83.

43. Zhang J, Yang G, Wen Y, Liu S, Li C, Yang R, et al. Intestinal microbiota are involved in the immunomodulatory activities of longan polysaccharide. Mol Nutr Food Res. 2017;61(11):1. https://doi.org/10.1002/mnfr.201700466.

44. Rios-Covian D, Cuesta I, Alvarez-Buylla JR, Ruas-Madiedo P, Gueimonde M, de Los Reyes-Gavilan CG. Bacteroides fragilis metabolises exopolysaccharides produced by bifidobacteria. BMC Microbiol. 2016;16(1): 150. https://doi.org/10.1186/s12866-016-0773-9.

45. Hu Y, Chen H, Li P, Li B, Cao L, Zhao C, et al. Analysis of interactions between Endobiotics and human gut microbiota using in vitro Bath fermentation systems. J Vis Exp. 2019;150:1. https://doi.org/10.3791/59725.

46. Aoyama M, Kotani J, Usami M. Butyrate and propionate induced activated or non-activated neutrophil apoptosis via HDAC inhibitor activity but without activating GPR-41/GPR-43 pathways. Nutrition. 2010;26(6):653-61. https://doi.org/10.1016/j.nut.2009.07.006.

47. Chang PV, Hao L, Offermanns S, Medzhitov R. The microbial metabolite butyrate regulates intestinal macrophage function via histone deacetylase inhibition. Proc Natl Acad Sci U S A. 2014;111(6):2247-52. https://doi.org/10. 1073/pnas.1322269111

48. Zhang M, Sun $K$, Wu Y, Yang $Y$, Tso P, Wu Z. Interactions between intestinal microbiota and host immune response in inflammatory bowel disease. Front Immunol. 2017:8:942. https://doi.org/10.3389/fimmu.2017.00942.

49. Matsuzaki C, Hayakawa A, Matsumoto K, Katoh T, Yamamoto K, Hisa K. Exopolysaccharides produced by Leuconostoc mesenteroides strain NTM048 as an Immunostimulant to enhance the mucosal barrier and influence the systemic immune response. J Agric Food Chem. 2015;63(31): 7009-15.

50. Xiu L, Zhang HC, Hu ZP, Liang YC, Guo S, Yang M, et al. Immunostimulatory activity of exopolysaccharides from probiotic lactobacillus casei WXD030 strain as a novel adjuvant in vitro and in vivo. Food Agric Immunol. 2018; 29(1):1086-105.

51. Saadat YR, Khosroushahi AY, Gargari BP. A comprehensive review of anticancer, immunomodulatory and health beneficial effects of the lactic acid bacteria exopolysaccharides. Carbohydr Polym. 2019;217:79-89.

52. Jiang SM, Ma CC, Peng QN, Huo DX, Li W, Zhang JC. Microbial profile and genetic polymorphism of predominant species in some traditional fermented Seafoods of the Hainan area in China. Front Microbiol. 2019;10:564.

53. Ai LZ, Zhang H, Guo BH, Chen W, Wu ZJ, Wu Y. Preparation, partial characterization and bioactivity of exopolysaccharides from lactobacillus casei LC2W. Carbohydr Polym. 2008;74(3):353-7.

54. Kim MJ, Seo HN, Hwang TS, Lee SH, Park DH. Characterization of exopolysaccharide (EPS) produced by Weissella hellenica SKkimchi3 isolated from kimchi. J Microbiol. 2008;46(5):535-41. https://doi.org/10.1007/s12275008-0134-y.

55. Dubois M, Gilles KA, Hamilton JK, Rebers PA, Smith F. Colorimetric method for determination of sugars and related substances. Anal Chem. 1956;28(3): $350-6$.

56. He Q, Wang L, Wang F, Wang C, Tang C, Li Q, et al. Microbial fingerprinting detects intestinal microbiota dysbiosis in Zebrafish models with chemicallyinduced enterocolitis. BMC Microbiol. 2013;13:289.

57. Dethlefsen $L$, Relman DA. Incomplete recovery and individualized responses of the human distal gut microbiota to repeated antibiotic perturbation. Proc Natl Acad Sci U S A. 2011;108(Suppl 1):4554-61. https://doi.org/10. 1073/pnas.1000087107.

58. Tan L, Ju H, Li J. Extraction and determination of short-chain fatty acids in biological samples. Se Pu. 2006;24(1):81-7.

59. Caporaso JG, Kuczynski J, Stombaugh J, Bittinger K, Bushman FD, Costello EK, et al. QIIME allows analysis of high-throughput community sequencing data. Nat Methods. 2010;7(5):335-6. https://doi.org/10.1038/nmeth.f.303.

60. DeSantis TZ, Hugenholtz P, Larsen N, Rojas M, Brodie EL, Keller K, et al. Greengenes, a chimera-checked $16 \mathrm{~S}$ rRNA gene database and workbench compatible with ARB. Appl Environ Microbiol. 2006;72(7):5069-72. https:// doi.org/10.1128/AEM.03006-05.

61. Langille MG, Zaneveld J, Caporaso JG, McDonald D, Knights D, Reyes JA et al. Predictive functional profiling of microbial communities using $16 \mathrm{~S}$ rRNA marker gene sequences. Nat Biotechnol. 2013;31(9):814-21. https://doi. org/10.1038/nbt.2676.

62. Ito K, Murphy D. Application of ggplot2 to Pharmacometric graphics. CPT Pharmacometrics Syst Pharmacol. 2013;2:e79. https://doi.org/10.1038/psp. 2013.56.

63. Zapala MA, Schork NJ. Multivariate regression analysis of distance matrices for testing associations between gene expression patterns and related variables. Proc Natl Acad Sci U S A. 2006;103(51):19430-5. https://doi.org/10. 1073/pnas.0609333103.

64. Shannon P, Markiel A, Ozier O, Baliga NS, Wang JT, Ramage D, et al. Cytoscape: a software environment for integrated models of biomolecular interaction networks. Genome Res. 2003;13(11):2498-504. https://doi.org/10. 1101/gr.1239303.

\section{Publisher's Note}

Springer Nature remains neutral with regard to jurisdictional claims in published maps and institutional affiliations.

Ready to submit your research? Choose BMC and benefit from:

- fast, convenient online submission

- thorough peer review by experienced researchers in your field

- rapid publication on acceptance

- support for research data, including large and complex data types

- gold Open Access which fosters wider collaboration and increased citations

- maximum visibility for your research: over $100 \mathrm{M}$ website views per year

At $\mathrm{BMC}$, research is always in progress.

Learn more biomedcentral.com/submissions 